\title{
Test of shell-model interactions for nuclear structure calculations
}

\author{
M. Thoresen, ${ }^{1}$ D. C. Zheng, ${ }^{2}$ and B. R. Barrett ${ }^{1}$ \\ ${ }^{1}$ Department of Physics, University of Arizona, Tucson, Arizona 85721 \\ ${ }^{2}$ W.K. Kellogg Radiation Laboratory, California Institute of Technology, Pasadena, California 91125
}

(Received 19 December 1995)

\begin{abstract}
The binding energy and excitation spectra of ${ }^{6} \mathrm{Li}$ are calculated in a no-core shell-model space giving encouraging results. The results of this calculation are then treated as a theoretical experiment, against which different effective-interaction approximations are compared. In this way insight into the perturbation expansion for the effective interaction is obtained.
\end{abstract}

PACS number(s): 21.60.Cs, 21.10.Dr, 21.30.Fe, 27.20.+n

Recently no-core shell-model calculations have been performed [1,2], in which all nucleons are active, so there are no hole states. This approach avoids the use of shell-model effective interactions involving excitations from an inert core of nucleons (e.g., the core-polarization process), and thereby avoids the convergence problems of the standard effectiveinteraction approach [3].

In this paper we take advantage of the fact that no-core shell-model calculations have had considerable success in reproducing the energy spectra and other properties of light nuclei (i.e., $A \leqslant 7$ ) $[1]$ to propose a model for testing the convergence properties of shell-model effective interactions calculated in terms of perturbation theory. We first perform a no-core shell-model calculation for ${ }^{6} \mathrm{Li}$ in a large basis space and demonstrate that it reproduces the experimental binding energy and spectrum quite well. We then take the results of this no-core calculation to be those of a "theoretical experiment" against which the results of standard shell-model effective-interaction calculations can be compared. In this way we can gain insight into how the standard calculations succeed or fail, since we know what information went into the no-core calculations.

In a very large model space the effective two-body interaction among the nucleons should essentially be the $G$ matrix plus all folded diagrams derived from it. The $G$ matrix is simply the sum of all two-particle ladder excitations given by the expression

$$
G(\omega)=V+V \frac{Q}{\omega-H_{0}} G(\omega),
$$

where $V$ is the free nucleon-nucleon interaction. The starting energy $\omega$ represents the initial energy of the two nucleons in the medium. $H_{0}$ is the unperturbed Hamiltonian of the system, and $Q$ is the Pauli projection operator, which projects onto two-particle states that are not already occupied. In this study the Nijmegen II potential [4] is used for the nucleonnucleon interaction.

In an earlier investigation Zheng et al. [5] showed that in no-core calculations the sum of all folded diagrams can be reasonably approximated by calculating the two-body matrix elements of $G$ for different starting energies according to the energies of the two-particle states. The approximation suggested by them was

$$
\omega=\epsilon_{a}+\epsilon_{b}+\Delta,
$$

where $\epsilon_{i}$ is the harmonic-oscillator single-particle energy for the state $i$ and $\Delta$ represents the interaction energy between the particles $a$ and $b$. In general, $\Delta$ is state dependent, but Zheng et al. [5] found that good results could be obtained using a constant value for $\Delta$. In a later work Zheng et al. [1] found that $G$ plus all of its folded diagrams tends to overbind light nuclei, due to the omission of higher-order many-body effects, if the model space is not sufficiently large. Consequently, they decided to treat $\Delta$ as a parameter to obtain the correct binding energy for the model space utilized. In this way $\Delta$ also serves as a term to treat effectively the omitted many-body effects. In this study $\Delta$ is chosen to be -25 $\mathrm{MeV}$, so as to match the ground-state energy of ${ }^{6} \mathrm{Li}$ for the no-core calculation having a model space of eight major shells. This same $\Delta$ is used to determine $\omega$ in all other model spaces studied in this paper.

In the no-core calculations the $G$-matrix elements are calculated for model spaces containing all two-particle states with unperturbed energies up to $4 \hbar \Omega, 6 \hbar \Omega$, and $8 \hbar \Omega$, corresponding to excitations of $2 \hbar \Omega, 4 \hbar \Omega$, and $6 \hbar \Omega$ above the lowest-energy configuration of ${ }^{6} \mathrm{Li}$, respectively. Harmonicoscillator states are used for computing the two-body matrix elements of $G$, following the procedure of Barrett, Hewitt, and McCarthy [6], and a harmonic oscillator energy of $\hbar \Omega=14 \mathrm{MeV}$ is used. Using complete harmonic-oscillator spaces allows us to project out the spurious center-of-mass components in the wave functions [7].

The results for the no-core calculation depend on the size of the model space used, as shown in Fig. 1. In a $4 \hbar \Omega$ space the first excited $J=1, T=0$ state and the first $J=2, T=1$ state are inverted, while the other states are in the correct order. The correct ordering and general spacing of states is obtained in $6 \hbar \Omega$ and $8 \hbar \Omega$ spaces. There is a significant difference between the results obtained in the $4 \hbar \Omega$ and $6 \hbar \Omega$ spaces. The difference in the binding energies and excitation spectra between the $6 \hbar \Omega$ space and the $8 \hbar \Omega$ space is minimal. Figure 1 shows the convergence of the binding energy and spectrum as the size of the model space grows from $4 \hbar \Omega$ to $8 \hbar \Omega$. The no-core calculations of the excitation spectrum are seen to be converging as the size of the model space increases. The ordering of states matches the experimentally determined ordering for the six lowest states [8]. The next four calculated excited states are also shown. 


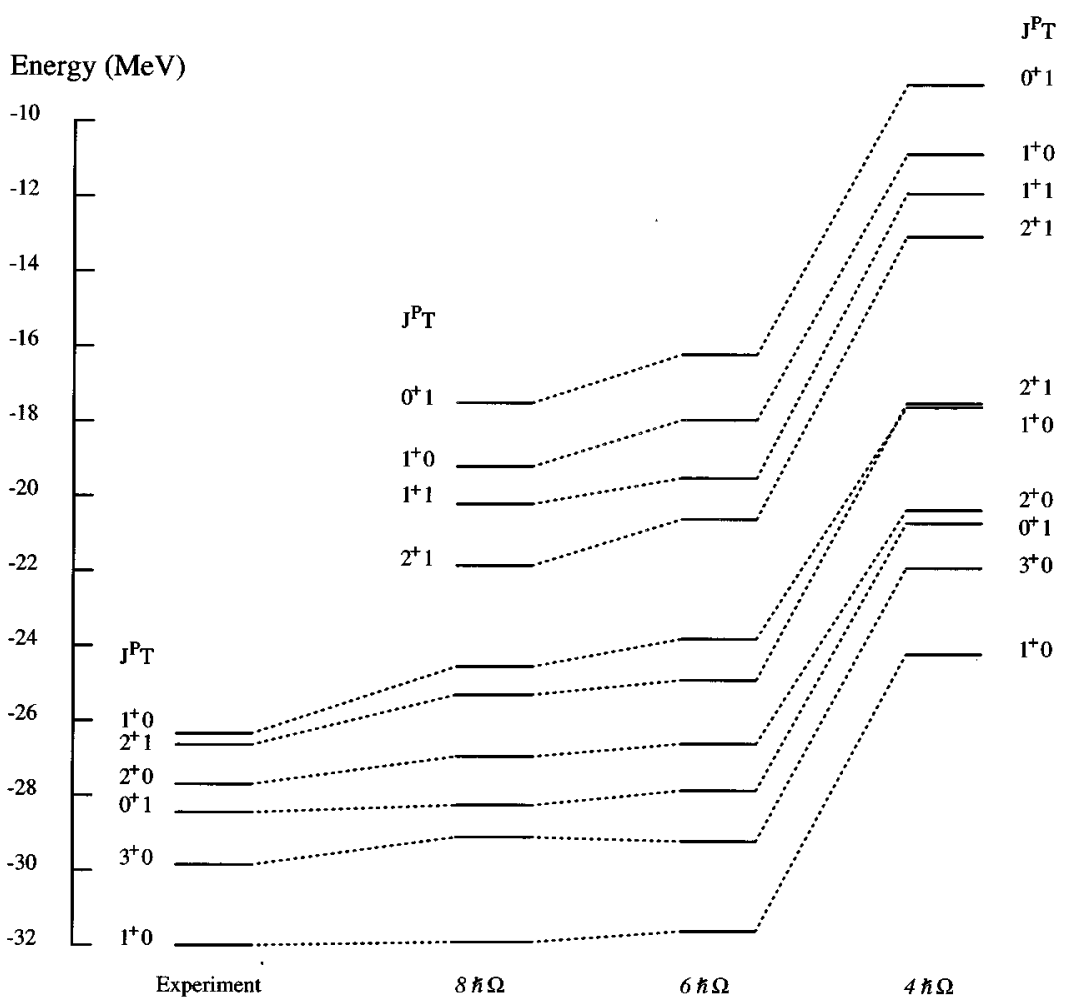

FIG. 1. No-core calculations of the binding energy and excitation spectrum of ${ }^{6} \mathrm{Li}$. Model spaces of $4 \hbar \Omega$, $6 \hbar \Omega$, and $8 \hbar \Omega$ are used. In all cases $\Delta$ is chosen to be $-25 \mathrm{MeV}$ and a harmonic oscillator energy of $\hbar \Omega=14 \mathrm{MeV}$ is used. These results are compared with the first six experimentally determined levels for ${ }^{6} \mathrm{Li}$ (Ref. [8]).

The perturbation-expansion diagrams for the effective interaction follow the work of Barrett and Kirson [9] and Kassis [10]. These diagrams involve interactions of the valence nucleons with particles from the core as well as interactions between the valence particles themselves. The order of a given perturbation diagram depends upon the number of interactions involved in that particular diagram. The first-order effective interaction is simply the $G$ matrix. Second-order terms for the effective interaction are obtained from all twobody diagrams that have two interactions involving $G$ and include the core-polarization, the two-particle-four-hole, and the second-order-ladder diagrams [9,11]. Third-order terms have three interactions involving $G$ and include the standard diagrams [9] as well as third-order-ladder diagrams [11] and folded diagrams. The total third-order effective interaction is taken to be the sum of all first-, second- and third-order diagrams.
The perturbation-theory calculation of the effective interaction begins by calculating the effective single-particle energies, using the same two-body $G$-matrix elements as in the $8 \hbar \Omega$ no-core calculation. The single-particle energies used in evaluating the denominators of the two-body diagrams are calculated using a second order perturbation expansion that involves evaluating the three one-body diagrams shown in Fig. 2. The total single-particle energy to second order is

$$
E_{a}^{\mathrm{total}}=E_{a}^{1^{\mathrm{st}}}+E_{a}^{2 p-1 h}+E_{a}^{3 p-2 h}
$$

The effective Hamiltonian, which includes one- and twobody terms, is evaluated to third order in the perturbation expansion. All terms contributing to the two-body effective interaction are computed with the same $G$ matrix elements and starting energy used in the no-core calculation involving eight major shells, and intermediate excitations of up to

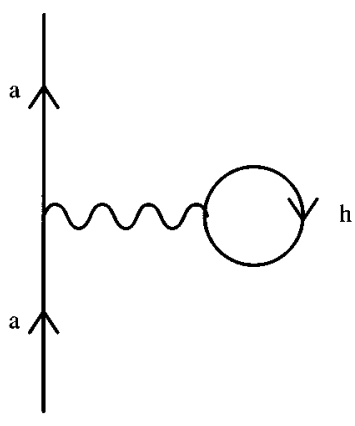

(a)

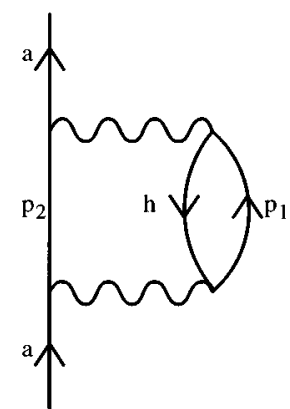

(b)

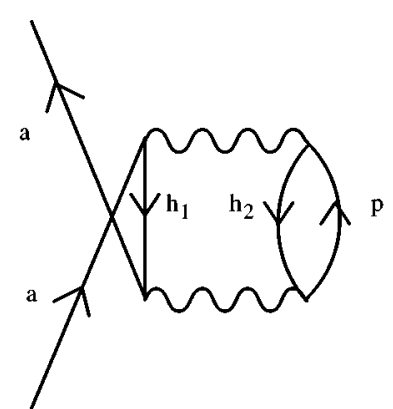

(c)

FIG. 2. One-body diagrams used in determining the single-particle energies to second order: (a) is the first-order, in $G$, diagram, (b) is the second-order two-particle-one-hole diagram, and (c) is the second-order three-particle-two-hole diagram. 


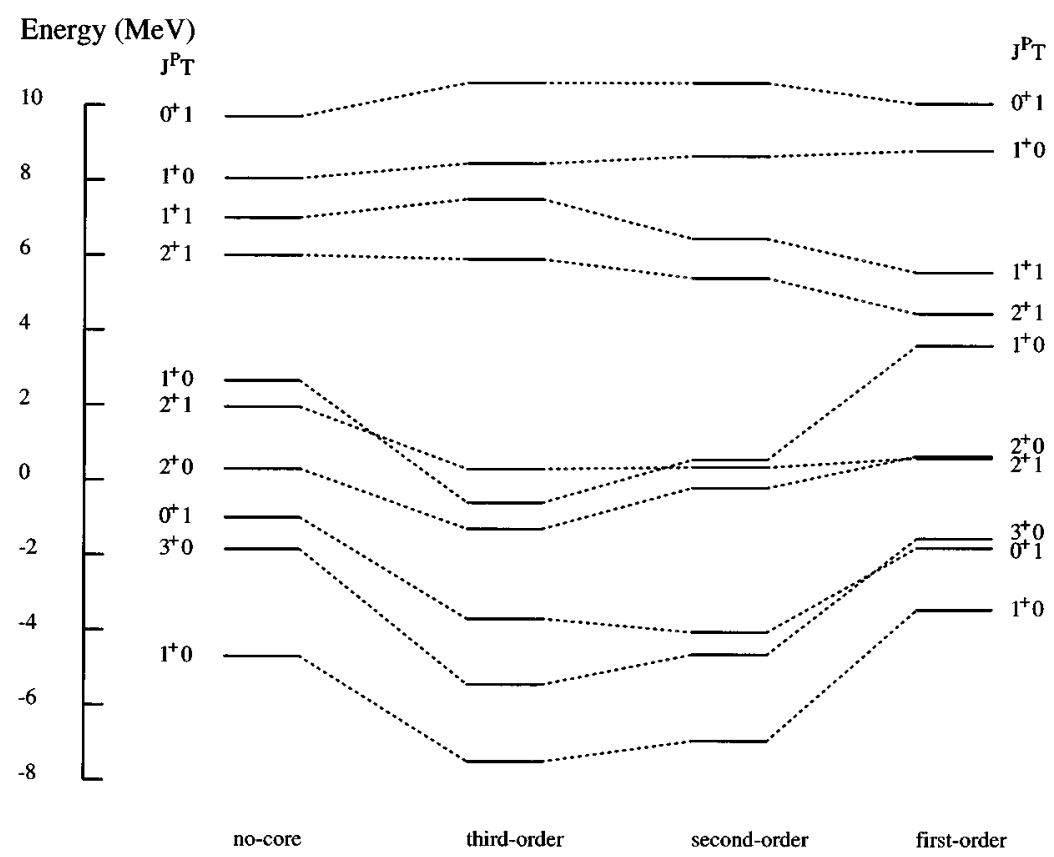

FIG. 3. Comparison of the order-by-order perturbation expansion results up to third order in $G$ with the no-core results. The ground-state energy for the no-core calculation is taken to be the Coulomb-corrected binding energy of ${ }^{6} \mathrm{Li}$ relative to the binding energy of ${ }^{4} \mathrm{He}$, which is $-4.700 \mathrm{MeV}$ (Ref. [12]). All calculations are done with the same $G$-matrix elements as used in the $8 \hbar \Omega$ no-core calculation with intermediate-state excitations up to $6 \hbar \Omega$. The model space, in which the effective Hamiltonian is diagonalized, is only the $0 p$ shell.

$6 \hbar \Omega$ are allowed in calculating all second- and third-order terms. This effective two-body interaction replaces the simple $G$-matrix in the final perturbation-expansion calculation of the energy levels of ${ }^{6} \mathrm{Li}$. Third-order terms contributing to the effective single-particle energies were calculated for the two $0 p$ states and were determined to be $4.652 \mathrm{MeV}$ for the $0 p_{1 / 2}$ state and $1.425 \mathrm{MeV}$ for the $0 p_{3 / 2}$ state. In general, the sum of all 16 third-order terms was small, being only $8 \%$ of the sum of the second-order terms on average. Consequently, it was decided not to calculate the third-order contributions to the single-particle energies used in calculating the effective two-body interaction. Finally, the energy spectrum of ${ }^{6} \mathrm{Li}$ is determined by diagonalizing the computed single-particle energies and the effective two-body interaction in the $0 p$ shell.

The no-core calculation in the full $8 \hbar \Omega$ model space is used as the "theoretical experiment" against which the perturbation-theory results are compared, as shown in Fig. 3. The ground-state energy for the no-core calculation is taken to be the Coulomb-corrected binding energy of ${ }^{6} \mathrm{Li}$ relative to the binding energy of ${ }^{4} \mathrm{He}$ which is $-4.700 \mathrm{MeV}$ [12]. It is seen that for the second- and third-order calculations the general ordering and spacing of the states is correct, with the exception of the first excited $J=1, T=0$ state being pushed below the first $J=2, T=1$ state in the third-order calculation. The spacing of states will be affected by states above them with the same $J$ and $T$ values. The first excited $(J=0$, $T=1),(J=2, T=1)$, and $(J=1, T=0)$ states are all pushed down by their respective higher-lying excited states. In most cases the third-order effect is small compared to first- and second-order effects. It can also be seen that as more diagrams are included, by going to higher order in $G$, the $T=0$ states, in general, become more attractive, while the $T=1$ states become more repulsive, which has been shown to be the case for a wide range of nuclei [13].
Figure 4 shows the results for the sum to third order in $G$ as the allowed intermediate-state excitations increase from $2 \hbar \Omega$ to $6 \hbar \Omega$. The third-order perturbation expansion for the effective interaction becomes more attractive as greater intermediate-state excitations are allowed. When compared with the no-core calculation in the same model space, the perturbation expansion in $G$ for intermediate-state excitations of $6 \hbar \Omega$ shows greater attraction for low-lying states than the no-core calculations with the same allowed excitations. This discrepancy cannot be due to omitted higher-lying excitations, because the original no-core calculation only involved excitations up to $6 \hbar \Omega$. Consequently, this difference is probably due to missing higher-order folded diagrams, which may account for neglected many-body forces. These many-body forces are overall repulsive and would serve to bring the perturbation spectrum back up towards agreement with the no-core spectrum [14].

To summarize, a large basis-space no-core calculation with all nucleons active gives a good reproduction of the known spectrum of ${ }^{6} \mathrm{Li}$. We can use the results of the no-core calculation as those of a theoretical experiment against which we can compare standard perturbation-theory calculations for the effective interaction. Such a comparison is useful because we know the input to the no-core calculation and how it differs from the calculation for the effective interaction. We find results consistent with previous effectiveinteraction investigations [13], namely: (1) the second-order terms (e.g., the core-polarization process) generally lead to attraction for $T=0$ states and repulsion for $T=1$ states, and (2) the third-order $T=0$ contributions are sizable, while the third-order $T=1$ contributions are small and more or less negligible.

Because we use the same harmonic-oscillator basis for both the no-core and effective-interaction calculations, we know that differences in the calculations are not due to 


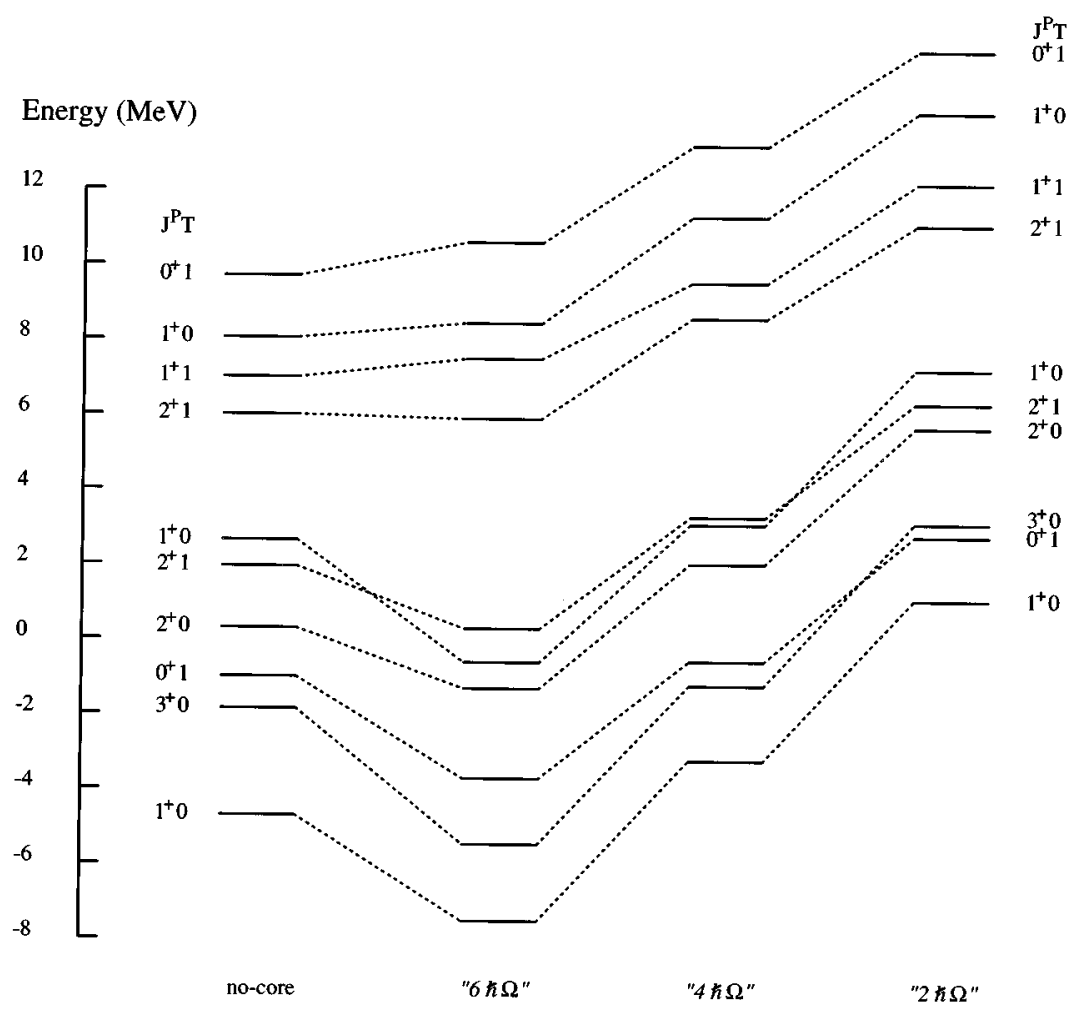

FIG. 4. Comparison of the third-order perturbation-expansion results, for allowed intermediate-state excitations of $2 \hbar \Omega$, $4 \hbar \Omega$, and $6 \hbar \Omega$ above the ground state, to the $8 \hbar \Omega$ no-core result. Again, the ground-state energy for the no-core calculation is taken to be $-4.700 \mathrm{MeV}$. All calculations are done with the same $G$-matrix elements as used in the $8 \hbar \Omega$ no-core calculation.

mean-field effects. Also, because the no-core calculation is truncated at $6 \hbar \Omega$ excitations above the ground-state configuration, we know that discrepancies in the effectiveinteraction calculation are not due to omitted higher-lying excitations. In fact, the only major difference between the two calculations is the treatment of effective many-body forces. (There are no real many-body forces in either calculation.) The no-core calculation has effective three and higher-body forces, due to diagonalization of $G$ in the space of six nucleons being active for excitations up to $6 \hbar \Omega$. The effective interaction is only for two-body interactions, which are diagonalized in the $0 p$ shell for two active nucleons. Consequently, the results of our calculations clearly demonstrate that the failure of our effective interaction to reproduce the no-core results is due to the omission of these effective many-body interactions, which are obviously repulsive for $T=0$, because the low-lying states are too attractive compared with the no-core results. Because all the features of our effective-interaction results are the same as previous, standard effective-interaction investigations, we conclude that, without adequate treatment of effective many-body interactions, effective-interaction shell-model calculations will fail to accurately describe nuclear spectra. The disagreement will become worse as the number of valence nucleons increases, since the many-body effects will increase relative to the twobody effects.

This work was supported in part by the National Science Foundation Grant No. PHY93-21668.
[1] D.C. Zheng, J.P. Vary, and B.R. Barrett, Phys. Rev. C 50, 2841 (1994).

[2] D.C. Zheng, B.R. Barrett, L. Jaqua, J.P. Vary, and R.J. McCarthy, Phys. Rev. C 48, 1083 (1993).

[3] T.H. Schucan and H.A. Weidenmüller, Ann. Phys. (N.Y.) 73, 108 (1972); 76, 483 (1973).

[4] V.G.J. Stoks, R.A.M. Klomp, C.P.F. Terheggen, and J.J. de Swart, Phys. Rev. C 49, 2950 (1994).

[5] D.C. Zheng, B.R. Barrett, J.P. Vary, and R.J. McCarthy, Phys. Rev. C 49, 1999 (1994).

[6] B.R. Barrett, R.G.L. Hewitt, and R.J. McCarthy, Phys. Rev. C 3, 1137 (1971).
[7] A.G.M. van Hees and P.W.M. Glaudemans, Nucl. Phys. A396, 105 (1983).

[8] F. Ajzenberg-Selove, Nucl. Phys. A490, 1 (1988).

[9] B.R. Barrett and M.W. Kirson, Nucl. Phys. A148, 145 (1970).

[10] N.I. Kassis, Nucl. Phys. A194, 205 (1972).

[11] B.R. Barrett, Nucl. Phys. A221, 299 (1974).

[12] E.K. Warburton and B.A. Brown, Phys. Rev. C 46, 923 (1992).

[13] M. Hjorth-Jensen, H. Müther, E. Osnes, and A. Ploos, “Comparison of the Effective Interaction to Various Orders in Different Mass Regions," ECT* Report, 1995.

[14] D.C. Zheng, B.R. Barrett, J.P. Vary, and H. Müther, Phys. Rev. C 51, 2471 (1995). 